\title{
Optimization of Charge Pumping Technique in Polysilicon TFTs for Geometric Effect Elimination and Trap State Density Extraction
}

\author{
Lei Lu, Mingxiang Wang ${ }^{*}$, Man Wong ${ }^{1}$ \\ Dept. of Microelectronics, Soochow University, Suzhou 215021, P. R. China \\ ${ }^{1}$ Dept. of Electronic and Computer Engineering, the Hong Kong University of Science and Technology, Hong Kong \\ *Email: mingxiang_wang@suda.edu.cn
}

\begin{abstract}
Charge pumping (CP) technique is optimized to minimize the geometric component in the $\mathrm{CP}$ current in polysilicon TFTs, by taking into account the pulse waveform and its transition times. Based on the optimization, ideal CP curves similar to those in MOSFETs are obtained. Important information on the trap state density of polysilicon TFTs, i.e., the mean value as well as the energy distribution within the band-gap, can be reliably extracted in different ways.
\end{abstract}

\section{Introduction}

Polysilicon TFTs have been widely used in active-matrix displays [1]. Charge pumping (CP) technique has been used as a powerful and reliable method for interface state density extraction in MOSFETs [2]. So far, a few works have been reported to characterize the trap properties of polysilicon TFTs by using CP [3-10]. It has also been employed to investigate the degradation of polysilicon TFTs [11, 12]. However, unlike in MOSFETs, CP never becomes a standard characterization technique for polysilicon TFTs, because it is still not sufficiently reliable and some ill-understood phenomena are not clarified $[3,5,7,12]$, among which the geometric effect is an important one.

In MOSFETs CP measurement, at the pulse rising edge, some free electrons which do not have enough time to flow back to the source/drain will be recombined with holes from the substrate. Similar process also happens at the pulse falling edge for holes. They also contribute to the recombined charge per cycle $\left(Q_{s s}\right)$ besides the "standard" $\mathrm{CP}$ mechanism, and provide an additional component in the CP current $\left(I_{c p}\right)$, i.e., the geometric component current $\left(I_{g e o}\right)$ [2]. Geometric effect in MOSFETs was found to depend on device $\mathrm{W} / \mathrm{L}$, pulse waveform, pulse transition time, etc [2]. In this paper, $I_{g e o}$ in polysilicon TFTs is eliminated (or minimized) by applying optimized pulse waveform and transition times, which makes CP quantitatively more reliable for the determination of the trap state density $\left(D_{t}\right)$.

\section{Experimental}

The experimental set-up for measuring $I_{c p}$ is illustrated

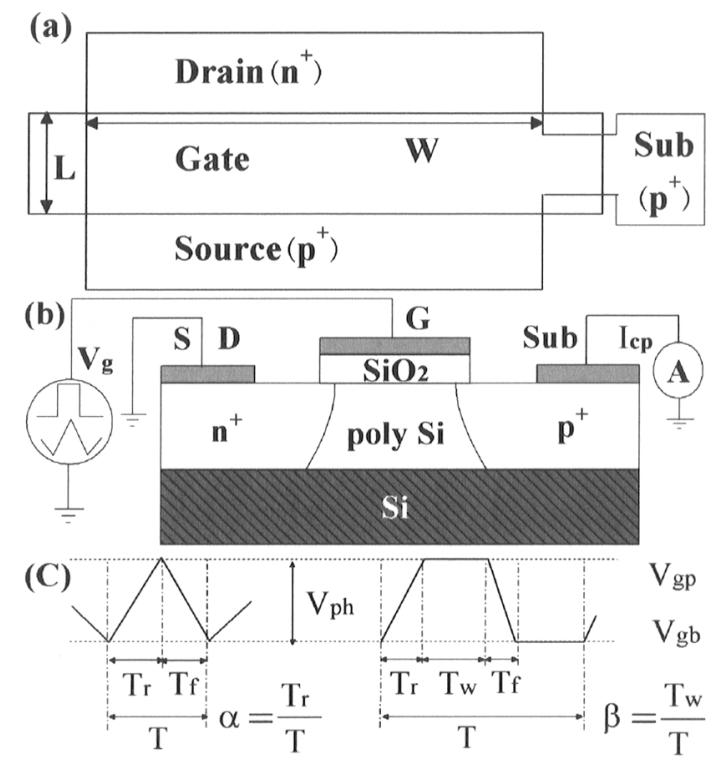

Figure 1. (a) Plane of the device. (b) Cross-sectional view of the device and the schematic diagram of the measurement. (c) Pulse parameters definition.

in Fig.1 for n-channel polysilicon TFTs with $\mathrm{W} / \mathrm{L}=30 / 10 \mu \mathrm{m}$. Polysilicon is formed by the solution based metal-induced crystallization technique and is in Fig.1 for n-channel polysilicon TFTs with $\mathrm{W} / \mathrm{L}=30 / 10 \mu \mathrm{m}$. Polysilicon is formed by the solution based metal-induced crystallization technique and is later recrystallized at $900^{\circ} \mathrm{C}$ [13]. The plane view (a) and cross-sectional view along the channel width direction (b) are shown in Fig.1. The source and drain are grounded. The $\mathrm{p}^{+}$diffusion is the polysilicon substrate terminal, from which $I_{c p}$ is measured by applying the square or triangular gate pulses at room temperature. The gate pulse parameters are defined in Fig.lc, including the pulse height $\left(V_{p h}=3 \sim 6 \mathrm{~V}\right)$, base voltage $\left(V_{g b}=-10 \sim 0 \mathrm{~V}\right)$, peak voltage $\left(V_{g p}=-5 \sim 7 \mathrm{~V}\right)$, period $(T=10 \sim 1000 \mu \mathrm{s})$, rising time $\left(T_{r}=0.1 \sim 7 \mu \mathrm{s}\right)$, falling time $\left(T_{f}=0.1 \sim 100 \mu \mathrm{s}\right)$ and the duty ratio for the triangular or square pulses $(\alpha, \beta=0.5)$. The $\mathrm{CP}$ measurement is performed in two ways: keep a constant $V_{p h}$ while increasing $V_{g b}$ (method A) or keep a constant $V_{g p}$ while increasing $V_{p h}(\operatorname{method} \mathrm{B})$. 


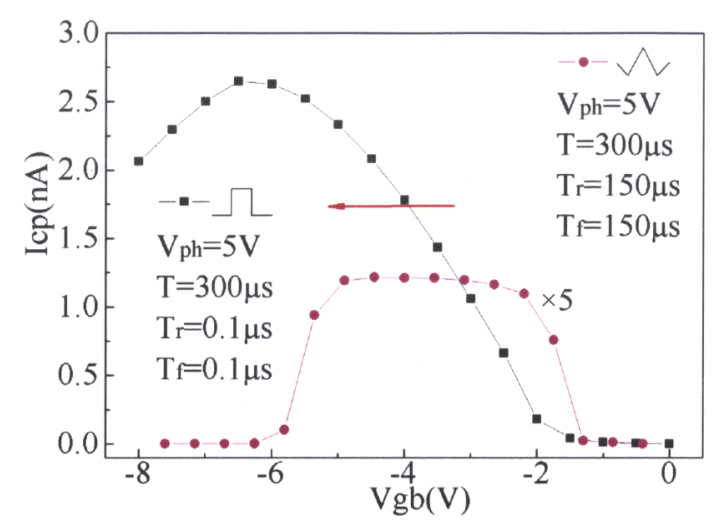

Figure 2. $I_{c p}$ versus $V_{g b}$ for square and triangular gate pulses using method A. $I_{c p}$ for triangular pulses is multiplied by 5 .

\section{CP optimization for geometric effect elimination}

In MOSFETs the geometric effect is supposed to be minimized by using device having W/L $>>1$ [2]. In Fig.2, the $I_{c p}$ is shown with $\mathrm{W} / \mathrm{L}=30 / 10 \mu \mathrm{m}$ when using the method A for square and triangular pulses. One can notice that the $I_{c p}$ peak value obtained by applying triangular pulses is much smaller than that of square pulses. Furthermore, the $I_{c p}$ under square pulses is asymmetric and largely shifted toward the negative, while that under triangular pulses is well shaped and is similar to those for MOSFETs. It clearly demonstrates that the geometric effect in polysilicon TFTs can not be suppressed by increasing W/L. A previous study [5] proposed that the substrate resistance of polysilicon TFTs is much higher than that of MOSFETs due to the presence of grain boundaries, resulting in a large $I_{g e o}$ in $I_{c p}$. The transition times $\left(T_{r}=T_{f}\right)$ of triangular pulses are much longer than those of square pulses, so electrons (or holes) can have enough time to flow back to the source/drain (or substrate) before recombination occurs and therefore $I_{g e o}$ is minimized.

In Fig.3, by increasing transition times $\left(T_{r}=T_{f}\right)$ of square pulses from $0.1 \sim 7 \mu \mathrm{s}, I_{c p}$ is continuously decreased. In the meanwhile, the distortion of $I_{c p}$ curve by $I_{g e o}$ in the negative $V_{g b}$ region becomes less and less significant. A typically symmetrical $I_{c p}$ curve is obtained when transition times is longer than $1 \mu \mathrm{s}$, with a well-defined saturated $I_{c p}$ values. It clearly demonstrates that $I_{g e o}$ is minimized or eliminated by increasing the pulse transition times. It can be well understood by the previously proposed mechanism [5]. To further clarify the influence of $T_{r}$ and $T_{f}$, also shown in Fig.3, $I_{c p}$ measured under symmetric pulses (open symbols) are compared with those under asymmetric ones (solid symbols). Interestingly, $I_{c p}$ curves under the same $T_{r}$ of either 0.1 or $1 \mu \mathrm{s}$ but very different $T_{f}$ coincide with each other; while those curves under the same $T_{f}$ of either 0.1

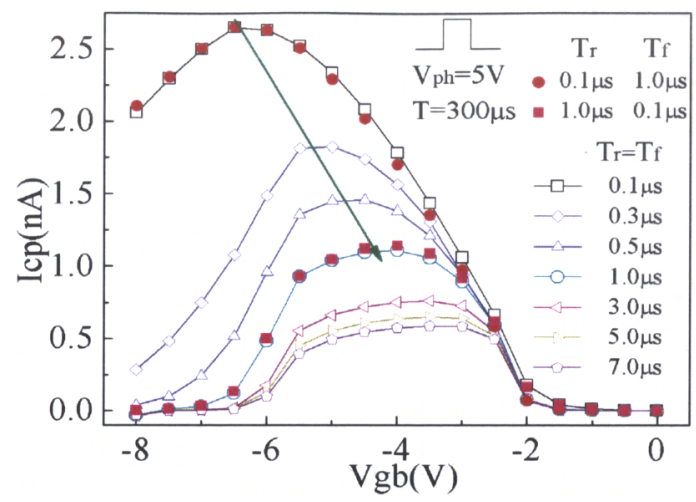

Figure 3. $I_{c p}$ versus $V_{g b}$ for square gate pulse with different $T_{r}$ and $T_{f}$ using method A.

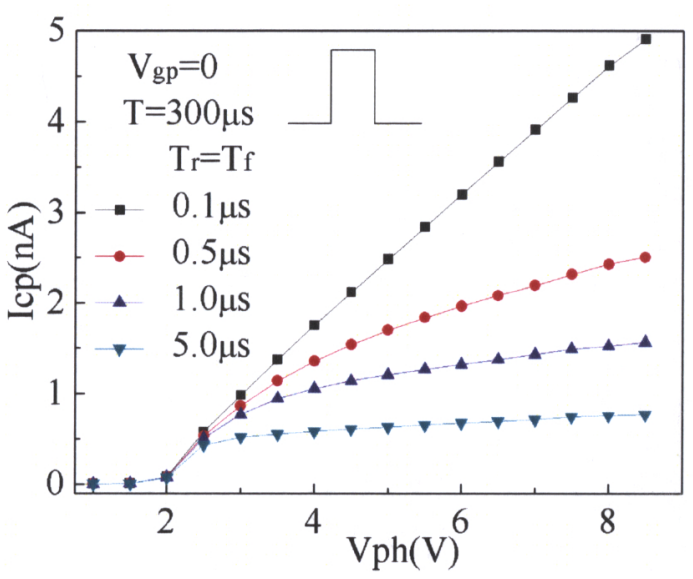

Figure 4. $I_{c p}$ versus $V_{p h}$ for square gate pulse with different $T_{r}$ and $T_{f}$ using method B.

or $1 \mu$ s but different $T_{r}$ are quite different. It means that the observed $I_{c p}$ is controlled by $T_{r}$ only.

In polysilicon TFTs there is no substrate bottom contact, a side contact has to be used for the $I_{c p}$ measurement, as shown in Fig.1a. Since $\mathrm{W}>>\mathrm{L}$, during the pulse rising edge, time needed for the accumulated holes to transit back to the substrate must be much longer than that of inverted electrons to drift back to the source/drain during the pulse falling edge. Furthermore, electrons can quickly drift along the low resistance inversion channel, while holes must diffuse through the substrate bulk to the side contact. Hence, if $T_{r}$ is not long enough, recombination of the remaining holes in the channel will contribute to the $I_{g e o}$ of polysilicon TFTs. From Fig.3, transit time of holes can be estimated as $>\sim 1 \mu$ s while that of electrons should be less than $0.1 \mu \mathrm{s}$.

Shown in Fig. 4 are $I_{c p}$ curves measured by method B using square pulse. $I_{c p}$ continuously decreases by increasing $T_{r}=T_{f}$. When the transition time is long enough $(>=1 \mu \mathrm{s}), I_{\text {geo }}$ becomes significantly suppressed and typical $I_{c p}$ saturation is observed, agreeing well with 


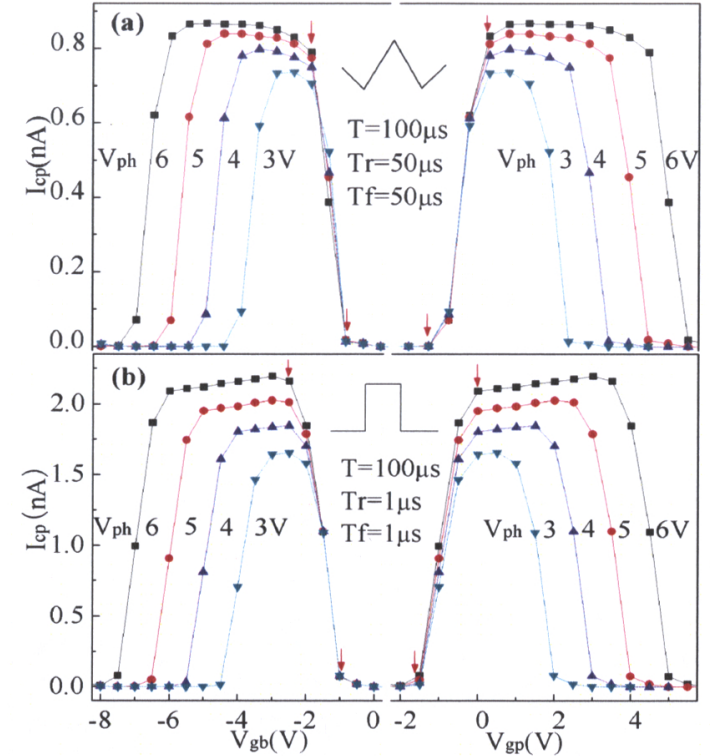

Figure 5. $I_{c p}$ as functions of $V_{g b}$ and $V_{g p}$ for triangular (a) and square pulse (b) with different $V_{p h}$ using method A.

the result in Fig.3. As for the $I_{c p}$ curve of $T_{r}=T_{f}=0.1 \mu \mathrm{s}$, an apparent linear dependence is obtained. In MOSFETs, it is known that $I_{\text {geo }}$ follows the following formula [2]:

$$
I_{g e o}=\delta \cdot f A_{g} C_{o x}\left(V_{g}-V_{t}\right)
$$

where $f, A_{g}, C_{o x}, V_{g}, V_{t}$ and $\delta$ are the pulse frequency, gate area, the gate oxide capacitance per unit area, the gate voltage, the device threshold voltage and the proportion of recombined carriers, respectively. From the slope of $I_{c p}$ dependence $\delta$ can be determined to be 0.89 . It indicates that most holes remain in the channel after pulse rising edge and are subsequently recombined by the inversion electrons.

Shown in Fig.5 are $I_{c p}$ curves measured by optimized waveform for (a) triangular and (b) square pulses. $I_{c p}$ is plotted against $V_{g b}$ in the left and $V_{g p}$ in the right. With $I_{\text {geo }}$ eliminated from the $I_{c p}$, ideally shaped CP curves with clear falling/rising edges and central flat regions are obtained under both conditions. Besides, the falling/rising edges $\left(V_{g b} \approx-1 \mathrm{~V}\right.$ and $V_{g p} \approx-1.5 \mathrm{~V}$, as indicated) of two measurement conditions coincide, so do the positions of central flat regions (between $V_{g p}>0$ and $V_{g b}<-2 \mathrm{~V}$, as indicated). The maximum $I_{c p}$ is seen to gradually increase with $V_{p h}$, which is in agreement with a previous $\mathrm{CP}$ model [2]. Compared to previous $\mathrm{CP}$ studies in polysilicon TFTs [3,7], the optimized $I_{c p}$ shown here is clearly more suitable for the $D_{t}$ extraction.

\section{4. $D_{t}$ extraction}

Three approaches have been proposed to extract $\bar{D}_{t}$

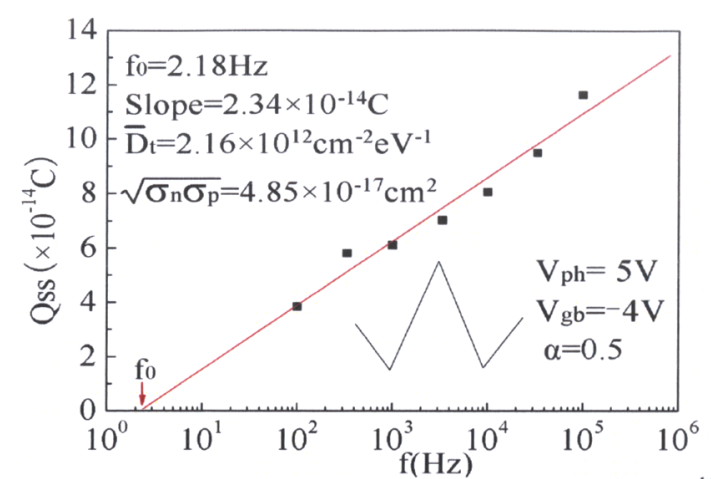

Figure 6. $Q_{s s}$ versus frequency used to determine $\left(\sigma_{n} \sigma_{p}\right)^{1 / 2}$ and $\bar{D}_{t}$.

or the energy distribution of $D_{t}$ using CP in MOSFETs [2]. In the first approach, $\bar{D}_{t}$ is obtained from the frequency dependence of $Q_{s s}$ by applying triangular pulses [2]. It allows the extraction of $\bar{D}_{t}$ without the need to know surface potential dependence on $V_{g}$. The semi-log plot of $Q_{s s}$ as a function of $f$ is shown in Fig.6. A straight line is obtained. Its slope and the intercept at $x$-axis $f_{o}$ can be used to determine $\bar{D}_{t}$ and $\left(\sigma_{n} \sigma_{p}\right)^{1 / 2}$ (mean capture cross section) by:

$$
\begin{aligned}
& \sqrt{\sigma_{n} \sigma_{p}}=\frac{1}{v_{t h} n_{i}} \cdot \frac{V_{p h}}{\left|V_{f b}-V_{t}\right|} \cdot \frac{f_{0}}{\sqrt{\alpha(1-\alpha)}} \\
& d Q_{s s} / d \ln f=2 q k T A_{g} \cdot \bar{D}_{t}
\end{aligned}
$$

where $\sigma_{n} / \sigma_{p}, v_{t h}, n_{i}, V_{f b}, k$ and $T$ are the electron/hole capture cross section, the thermal velocity, the intrinsic carrier density, the device flat band voltage, the Boltzman's constant and the temperature, respectively. $\bar{D}_{t}$ and $\left(\sigma_{n} \sigma_{p}\right)^{1 / 2}$ are determined to be $2.16 \times 10^{12} \mathrm{~cm}^{-2} \mathrm{eV}^{-1}$ and $4.85 \times 10^{-17} \mathrm{~cm}^{-2}$, respectively.

In the second approach, $D_{t}$ is obtained from the linear frequency dependence of $I_{c p}$ by applying square pulses [2, 3]. $\overline{D_{t}}$ is derived by the following equation:

$$
I_{c p}=2 q f A_{g} k T \cdot \bar{D}_{t} \ln \left(v_{t h} n_{i} \sqrt{\sigma_{n} \sigma_{p} T_{r} T_{f}} \frac{\left|V_{t}-V_{f b}\right|}{V_{p h}}\right)
$$

From Eq.2, $\left(\sigma_{n} \sigma_{p}\right)^{1 / 2}$ is obtained, on which $\bar{D}_{t}$ calculation from Eq.4 only weakly depends. $I_{c p}$ as a function of $f$ is plotted in Fig.7. One obtains a straight line, whose slope is used to extract $\bar{D}_{t}$ by Eq.4. $\bar{D}_{t}$ of $6.76 \times 10^{11} \mathrm{~cm}^{-2} \mathrm{eV}^{-1}$ is obtained.

In the third approach, energy distribution of $D_{t}$ is obtained from the transition time dependence of $I_{c p}$ by applying square pulses with different transition times [2, 4]. When varying $T_{f}$ and fixing $T_{r}$, one scans the energy range between the conduction band $\left(E_{c}\right)$ and the mid-gap $\left(E_{i}\right)$. $D_{t}$ energy distribution can be obtained as 


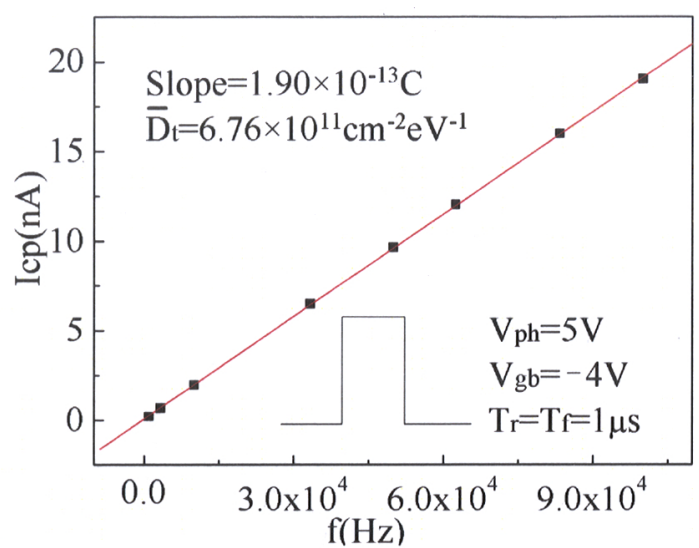

Figure 7. $I_{c p}$ versus frequency used to determine $\bar{D}_{t}$.

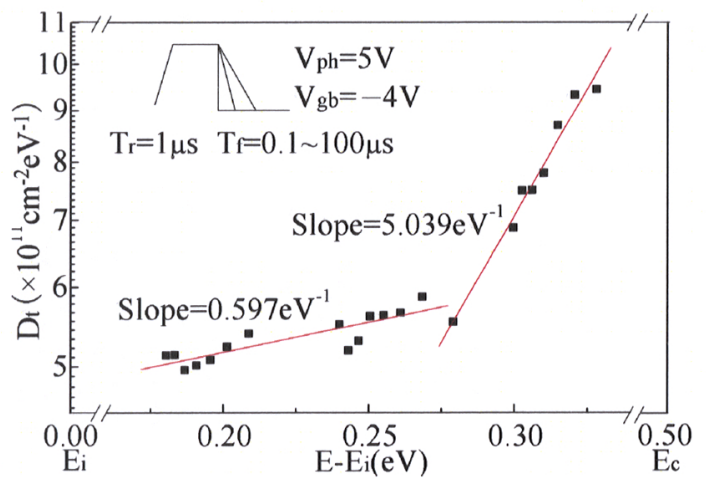

Figure 8. Dual exponential energy distribution of $D_{t}$.

follows:

$$
\begin{aligned}
& D_{t}(E)=-\frac{T_{f}}{q A_{g} k T f} \cdot \frac{d I_{c p}}{T_{f}} \\
& E-E_{i}=-k T \ln \left\lfloor v_{t h} \sigma_{n} n_{i} \cdot T_{f}\left|V_{f b}-V_{t}\right| / V_{p h}\right\rfloor
\end{aligned}
$$

Shown in Fig. 8 is the semi-log plot of the energy distribution obtained by Eq.5 and Eq.6. This dual exponential distribution has a small slope of $0.597 \mathrm{eV}^{-1}$ for deep states and a large slope of $5.039 \mathrm{eV}^{-1}$ for tail states, agreeing with the typical energy distribution of $D_{t}$ determined from other measurements $[14,15]$. The current result obtained by using optimized $\mathrm{CP}$ technique is in contrast to that in some previous $\mathrm{CP}$ studies for polysilicon TFTs, where irregular or distorted energy distribution of $D_{t}$ was obtained [5,6]. According to Eq.6, a broader range of energy distribution can still be achieved by changing the measurement temperature.

$D_{t}$ values in the latter two approaches agree well, which also agree with those of high temperature polysilicon TFTs in previous CP studies [3, 4, 6]. However, $D_{t}$ derived in the first approach is relatively larger, possibly because of different energy range in band-gap scanned by triangular pulses. A more elaborate study is needed to clarify such difference.

\section{Conclusions}

Optimized CP is used to minimize the $I_{g e o}$ in polysilicon TFTs, by taking into account the pulse waveform and transition time. Based on the optimization, ideal $I_{c p}$ curves are obtained. Furthermore, $D_{t}$ are extracted from three approaches, while $\bar{D}$, values agree well and typical dual exponential energy distribution of $D_{t}$ is obtained. It is confirmed with $I_{\text {geo }}$ minimized optimized CP is more reliable for $D_{t}$ extraction in polysilicon TFTs.

\section{Acknowledgments}

This work is supported by the National Natural Science Foundation of China.

\section{References}

[1] E. Persidis, H. Baur and F. Pieralisi et al., Solid-State Electron., Vol. 52, p. 455 (2008).

[2] G. Groeseneken, H. E. Maes and N. Beltran et al., IEEE Trans. Electron Devices, ED-31, p.42 (1984).

[3] M. Koyanagi and I-W. Wu et al., IEDM Tech. Dig., p.836 (1990).

[4] M. Koyanagi, Y. Baba and K. Hata et al., IEEE Electron Device Lett., Vol. 13, p.152 (1992).

[5] G. W. Lee, J. W. Lee and C. H. Han, Jpn. J. Appl. Phys., Vol. 38, p.2656 (1999).

[6] K. J. Kim and O. Kim, Jpn. J. Appl. Phys., Vol. 36, p.1394 (1997).

[7] N. S. Saks, S. Batra and M. Manning, Microelectronic Engineering, Vol. 28, p.379 (1995).

[8] A. Balasinski, J. Worley and J. Walters et al., IEEE Electron Device Lett., Vol. 16, p.460 (1995).

[9] K. J. Kim, W. K. Park and S. G. KIM, Solid-State Electron., Vol. 42, p.1897 (1998).

[10] O. Kim and K. J. Kim, Electronics Letters, Vol. 34, p.809 (1998).

[11] A. Balasinski and J. Worley et al., IEDM Tech. Dig., p.529 (1995).

[12] C. Y. Chen, M. W. Ma and W. C. Chen et al., IEEE Electron Device Lett., Vol. 29, p.165 (2008).

[13] B. Zhang, Z. Meng and S. Zhao et al., IEEE Trans. Electron Devices, Vol. 54, p. 1244 (2007).

[14] L. Pichon, A. Boukhenoufa and C. Cordier et al., J. Appl. Phys., Vol. 100, p. 54504-1 (2006).

[15] G. A. Armstrong, S. uppal and S. D. brotherton et al., Jpn. J. Appl. Phys., Vol. 37, p. 1721 (1998). 